\title{
Asymptomatic Bacteriuria in School-going Children in Lalitpur, Nepal
}

\author{
Bhuvan Saud ${ }^{1}$, Pravakar Sunuwar ${ }^{1}$, Govinda Paudel $^{1}$, Gunaraj Dhungana ${ }^{1}$, Vikram Shrestha ${ }^{1}$
}

${ }^{1}$ Department of Medical Laboratory Technology, Janamaitri Foundation Institute of Health Sciences (JFIHS), Hattiban, Lalitpur, Nepal

\section{Dear Editor,}

According to Infectious Diseases Society of America (IDSA), Asymptomatic Bacteriuria (ASB) is defined as the presence of one or more species of bacteria in urine at a count of $\geq 10^{5}$ colony-forming units $[\mathrm{CFU}] / \mathrm{mL}$ or $\geq 10^{8} \mathrm{CFU} / \mathrm{L}$, irrespective of the presence of pyuria, in the absence of signs or symptoms attributable to Urinary Tract Infection (UTI) ${ }^{1}$. Symptoms help a person to take treatment in time to reduce severe complications. Early detection of UTI in asymptomatic person can prevent renal damage, inflammation and other renal complications. Urinary tract may become infected by retrograde ascent of fecal-perianal bacteria, in medical setting through instrumentation, and involvement of urinary tract sometimes lead to systemic infection ${ }^{2}$. A total of 170 primary school children, 5 to 12 years of age, from Hattiban, Lalitipur were included for screening from April to October 2018. The students with the symptoms of UTI, under antibiotic therapy and those with anatomic abnormality in urinary tract were not included in this study. The study group involved 96 boys and 74 girls. The ethical approval was taken from Nepal Health Research Council, Kathmandu and written consent was taken from the parents of children. A clean catch midstream early morning urine sample was collected by the assistance of guardians in sterile wide mouth container and transported to the laboratory within an hour. Standard calibrated loop technique $(1 \mu \mathrm{L})$ was used to culture the sample. Colony count and identification of the isolates were done according to the Clinical Laboratory Standard Institute (CLSI) guidelines ${ }^{3}$. Count of $>10^{5} / \mathrm{mL}$ colony-forming units were read as significant growth and antimicrobial susceptibility testing was done according to KirbyBauer method. All culture positive samples were rechecked by collecting the second urine sample from children.
Out of the 170 children, $4.1 \%$ showed significant bacteriuria, all of which were from girl participants. The prominent isolates were Staphylococcus aureus (57.1\%), Escherichia coli (28.5\%) and Klebsiella pneumonia (14.3\%). All organisms were sensitive to Ciprofloxacin and Ceftriaxone. Three isolates (42.8\%) were Ampicillin resistant. Two of the isolates $(28.57 \%)$ were Erythromycin resistant. $14.2 \%$ of isolates were resistant to each of Cotrimoxazole, Nalidixic acid and Nitrofurantoin. $42.8 \%$ of the isolates were multidrug resistant and no Methicillin Resistant Staphylococcus aureus was found in this study.

Studies done in Nepal showed the prevalence to be in the range of $1.39 \%$ to $5.0 \%{ }^{4-5}$. Similar finding has also been reported in Egypt with a prevalence of $6.0 \%^{6}$, while study done in India showed $16.5 \%$ prevalence in school-going children ${ }^{7}$. Interestingly, similar to our study, all the studies discussed above showed higher prevalence in female children scompared to their male counterparts. Long term ASB may cause serious consequences in children health. Screening of ASB and awareness about hygiene can prevent the ascending UTI in female. Thus, screening for ASB in school going children helps early diagnosis, so that appropriate measures can be taken to prevent infection, pyelonephritis and late complication like chronic renal insufficiency. Hence we recommend routine screening for ASB in school going children so that unnecessary morbidity and mortality can be checked before it is too late.

\footnotetext{
Conflict of interest: None

Correspondence: Bhuvan Saud, Department of Medical Laboratory Technology, Janamaitri Foundation Institute of Health Sciences (JFIHS), Hattiban, Lalitpur, Nepal, GPO Box 8322; Email: link2bhuvan@gmail.com; Cell no.: +9779848724226
}

[Bangladesh Journal of Infectious Diseases, June 2019;6(1):2225 


\section{References}

1. Nicolle LE, Gupta K, Bradley SF, Colgan R, DeMuri GP, Drekonja D, et al. Clinical Practice Guideline for the Management of Asymptomatic Bacteriuria: 2019 Update by the Infectious Diseases Society of America. Clinical Infectious Diseases. 2019

2. Ma JF, Shortliffe LM. Urinary tract infection in children: etiology and epidemiology. Urologic Clinics. 2004;31(3):51726.

3. Clinical and Laboratory Standards Institute (CLSI) performance standards for antimicrobial disk diffusion susceptibility tests 19 th ed. approved standard. CLSI document
M100-S19. 2009;29(2011):M100-S21. Wayne, Pennsylvania. 4. Jha BK, Singh YI. Prevalence of asymptomatic bacteriuria in school going children in Pokhara valley. Kathmandu University Medical Journal. 2007;5(1):17-81.

5. Ghimire G, Singh YI. Asymptomatic Bacteriuria in School Going Children: A Study in Nepal. J Commun Dis 2016; 48(1):12-14.

6. Mohammed A, Abdelfattah M, Ibraheem A, Younes A. A study of asymptomatic bacteriuria in Egyptian school-going children. African health sciences. 2016;16(1):69-74

7. Kondapaneni S, Surpam R, Azaruddin M, Devi G. Screening for asymptomatic bacteriuria in school going children. Indian journal of public health. 2012;1;56(2):169 